\title{
Study on Synthesis of Maleic Anhydride Grafted Starch
}

\author{
Jian-Jiang SHANG ${ }^{1, a^{*}}$, Li-Na JIANG ${ }^{1, b}$, De-Qiang LI $^{2, c}$ and Xiao-Yan ZHU ${ }^{1, d}$ \\ ${ }^{1}$ Yili vocational and technology college, Xinjiang, Yining, 835000 \\ ${ }^{2}$ Xinjiang Agricultural University, Xinjiang, Urumqi, 830002 \\ axiaoxu_0614@yahoo.com.cn, ${ }^{b}$ sjj|xy@sohu.com, c475149588@qq.com, d893905883@qq.com \\ ${ }^{*}$ Corresponding author
}

Keywords: Maleic Anhydride, Starch, Modification.

\begin{abstract}
In order to improve the processing properties of starch, maleic anhydride grafted starch prepared by solution polymerization, With starch and maleic anhydride as raw materials. The structure of the material have been characterized by Fourier transform infrared spectrum(FTIR) and scanning electron microscopy (SEM). The results show that maleic anhydride grafted starch optimum conditions: ratio of starch and maleic anhydride dosage was 3:2.5, solvent water dosage was $1.0 \mathrm{~mL} / \mathrm{g}$, at $40{ }^{\circ} \mathrm{C}$, for $5 \mathrm{~h}$. The results showed that the grafting ratio was $47.27 \%$ and the grafting efficiency was $89.78 \%$ at this time.
\end{abstract}

\section{Introduction}

The physical properties of natural starch have restricted its application, such as unstable water sol, poor heat resistance and so on[1-2]. Therefore, in order to meet some specific needs, such as hot plastic extrusion process of starch, it is often required to chemically modify, to change its physical properties[3]. It is generally accepted that the starch derivatives with high degree of substitution can make the starch obtain better processing performance and application performance. However, due to the preparation conditions of starch derivatives with high degree of substitution are very complex, so the current commercial starch derivatives are usually only short alkyl chain and low degree of substitution of the product. In recent years, although the long alkyl chains and high degree of substitution of starch esters have been prepared under the conditions of environmental friendly conditions, the relevant preparation conditions need to be further improved[4-6]. In this study, the starch was modified by maleic anhydride, and the optimum synthesis conditions of maleic anhydride grafted starch was obtained.

\section{Material and Methods}

\section{Materials}

Maleic anhydride (AP) were obtained from Tianjin Tianxin Fine Chemicals Development Center, soluble starch (AP) from Tianjin Shengao Fine Chemicals Lit., acetone (AP) and pyridine (AP) from Shanghai Sanaisi chemical agent factory, and N,N - dimethylformamide(AP) from Tianjin Guangfu Fine Chemicals Lit., other reagents were analytical pure.

\section{Starch Gelatinization}

Take $3 \mathrm{~g}$ starch into the four neck flask, adding a certain amount of distilled water and $8 \mathrm{~mL} \mathrm{~N}$, $\mathrm{N}$ - dimethylformamide. The temperature is controlled below $55^{\circ} \mathrm{C}$. Heat insulation in 70 degrees 10-15 min, And then the temperature rose to 90-95 ${ }^{\circ} \mathrm{C}$, heat insulation 20-30 min.

\section{Preparation of Grafted Starch}

Cooling it to $50^{\circ} \mathrm{C}$ after starch gelatinization, it was slowly added to the maleic anhydride solution ( $7 \mathrm{~mL} \mathrm{~N}, \mathrm{~N}$ - dimethylformamide and $2 \mathrm{~mL}$ pyridine). Cooling product temperature to room temperature, adding hydrochloric acid to acid. The product was poured into $75 \%$ by volume ethanol 
aqueous solution. The precipitate with ethanol aqueous solution washing and filtering repeatedly, and then placed at $80{ }^{\circ} \mathrm{C}$ in vacuum drying box for drying, The crude product of the graft was maleic anhydride grafted starch.

\section{Purification of Product}

The starch maleic acid ester drying and weighing, with acetone as solvent, using soxhlet extractor extracting for $6 \mathrm{~h}$. The product obtained after extraction of the product was refined at $80^{\circ} \mathrm{C}$ in vacuum drying box. Weighing and recording after drying.

\section{Percent Grafting}

The percent grafting $(\mathrm{G})$ was calculated by Eq. 1, in which $\mathrm{W}_{0}$ is the weight of initial starch and $\mathrm{W}_{l}$ is the weight of maleic anhydride.

$$
\mathrm{G} \%=\frac{W_{2}}{W_{0}+W_{1}} \times 100 \%
$$

\section{Grafting Efficiency}

The grafting efficiency (GE) was calculated by Eq. 2, in which $\mathrm{W}_{2}$ is the weight of crude graft copolymer and $\mathrm{W}_{3}$ is the weight of pure starch graft copolymer.

$$
\mathrm{GE} \%=\frac{W_{3}}{W_{2}} \times 100 \%
$$

\section{Test and Characterization}

The IR spectra were recorded with a Fourier transform infrared spectroscopy (FT-IR) instrument in the in the 400-4000 $\mathrm{cm}^{-1}$ region. The micro morphology of samples was observed by JSM-6360LV scanning electron microscope (SEM).

\section{Result and Discussion}

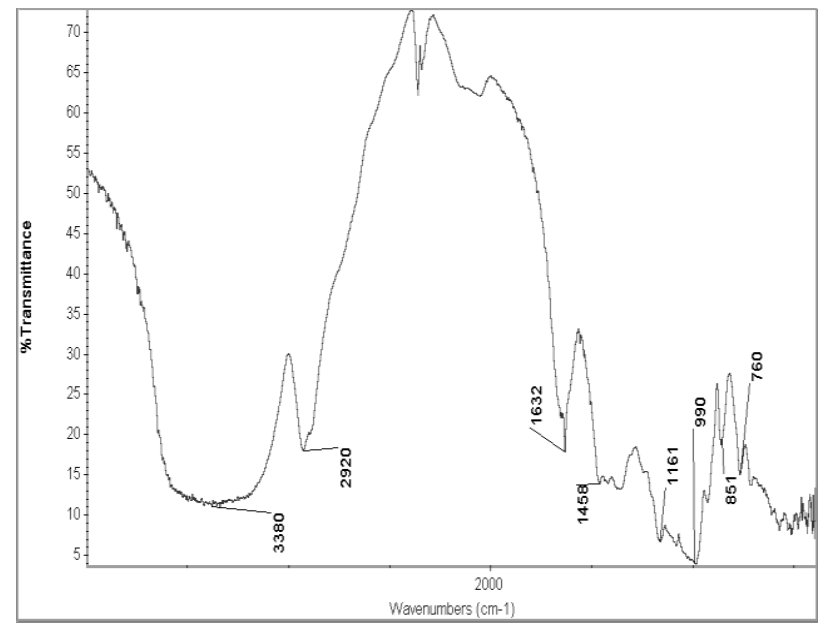

Fig. 1 IR spectra of starch 


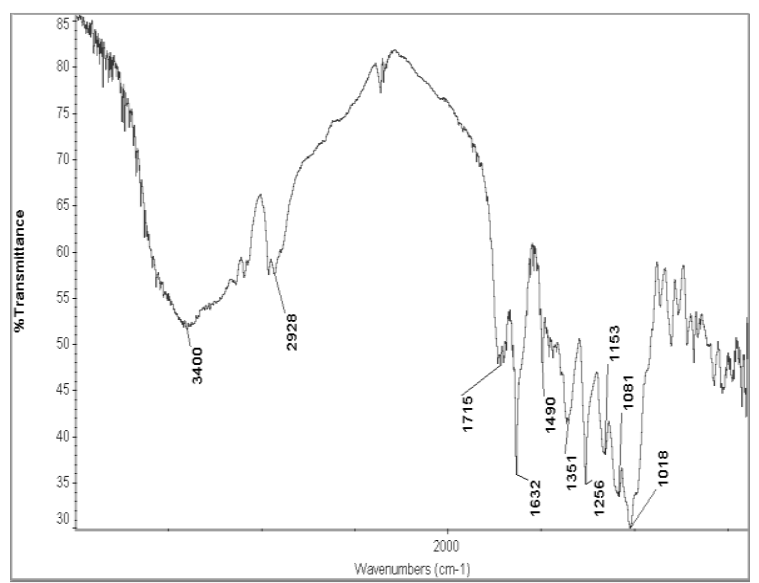

Fig. 2 IR spectra of maleic anhydride grafted starch

\section{IR Analysis}

The IR spectrum of starch was shown in Fig. 1. The peaks of about $851 \mathrm{~cm}^{-1}, 760 \mathrm{~cm}^{-1}$ and 580 $\mathrm{cm}^{-1}$ were the peaks of $-\mathrm{OH}$ rocking vibration absorption. The IR spectrum of starch was shown in Fig. 2. The peak of $\mathrm{C}=\mathrm{C}$ stretching vibration could be found about $1761 \mathrm{~cm}^{-1}$. The peak of $\mathrm{C}=\mathrm{C}$ stretching vibration could be found about $1761 \mathrm{~cm}^{-1}$. The peaks of $1761 \mathrm{~cm}^{-1}$ represented the stretching vibration of ester group. Starch has no absorption peak in the two position. The multi peak is strong and wide absorption peaks of starch C-O appeared from 1018 to $1153 \mathrm{~cm}^{-1} \mathrm{~cm}^{-1}$. Therefore, maleic anhydride was grafted starch and proved.

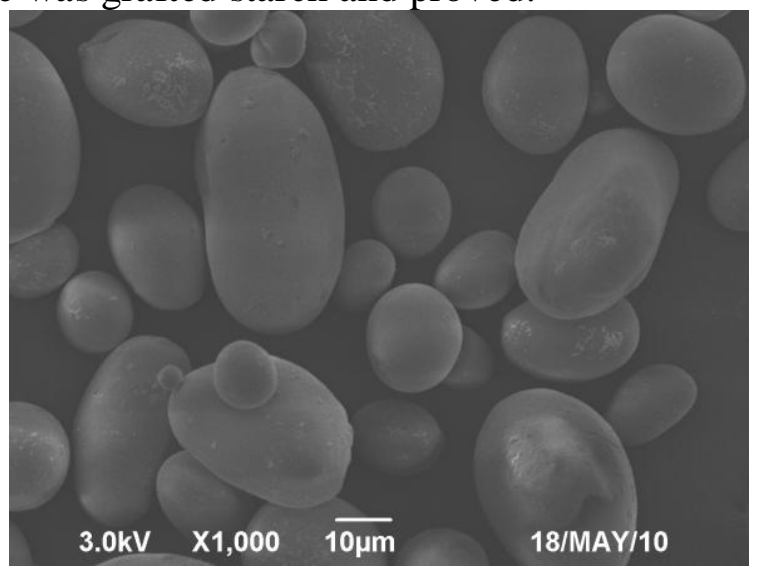

Fig. 3 SEM graph of starch

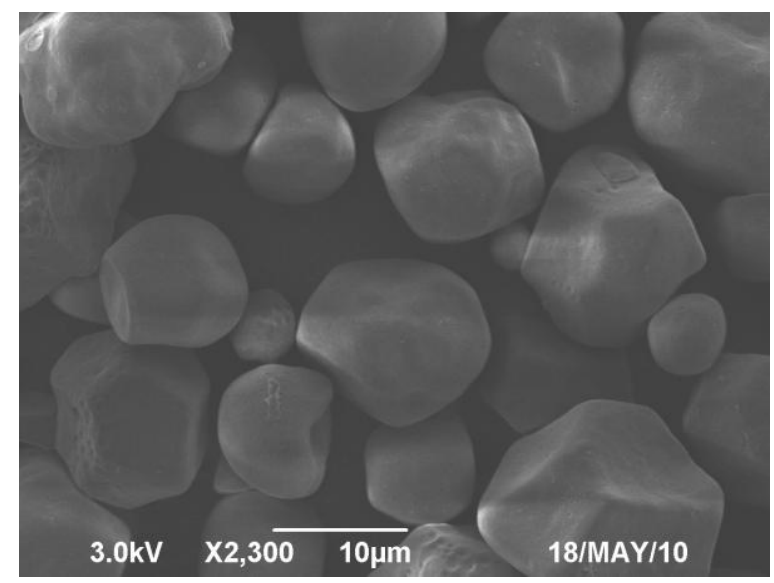

Fig. 4 SEM graph of maleic anhydride grafted starch 


\section{SEM Analysis}

Starch and starch after grafting modification were characterized by SEM, as shown in Fig. 3 and Fig. 4. Fig. 3 is the corn starch SEM graph, you can see the initial starch particles are solid circular or oval, smooth surface. Fig. 4 is the maleic anhydride starch SEM graph, compared with the initial starch, it can be seen: the appearance of the grafted starch after the appearance of a certain change, such as the surface is no longer smooth, outside by a certain "corrosion", loose structure, which shows that the starch and maleic anhydride grafting reaction.

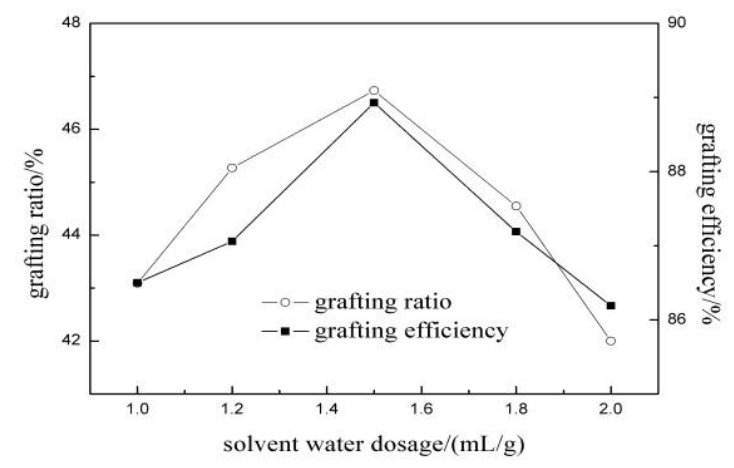

Fig. 5 Effect of the solvent water dosage on grafting ratio

\section{Effect of the Solvent Water Dosage}

Effect of water dosage on grafting ratio grafting ratio and grafting efficiency are shown in Fig. 5 . With the increase of solvent water dosage, starch grafting ratio increased gradually, the solvent water dosage of $1.5 \mathrm{~mL} / \mathrm{g}$, the grafting ratio reached the maximum. After that, with the increase of the solvent water dosage, the grafting ratio curve showed a downward trend. Add water solvent, the starch dissolved completely, keep the homogeneous reaction system, can promote the reaction. Maleic anhydride can be decomposed into maleic acid in water. Maleic acid activity in esterification reaction is low, leading to decreased the grafting ratio.

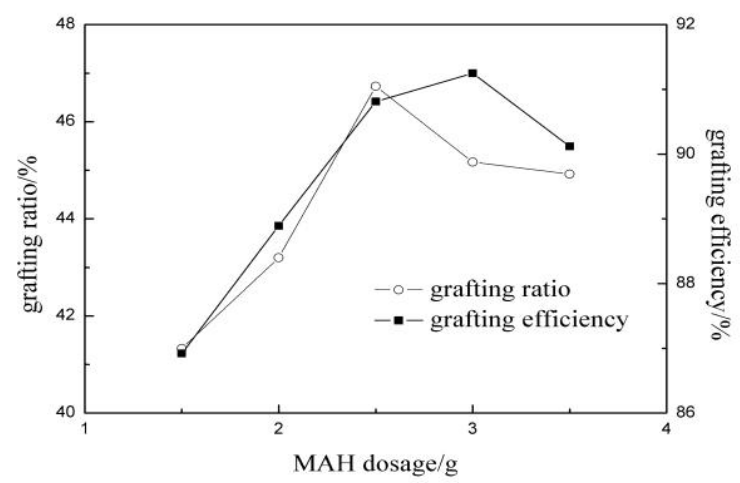

Fig. 6 Effect of the MAH dosage on grafting ratio

\section{Effect of the MAH Dosage}

Effect of MAH dosage on grafting ratio and grafting efficiency are shown in Fig. 6. When starch dosage was $3 \mathrm{~g}$, with the increase of MAH dosage, starch grafting ratio increased gradually. The ratio of starch dosage and MAH dosage was 3:2.5, the grafting ratio reached the maximum. After that, with the increase of MAH dosage, the grafting ratio curve showed a downward trend. 


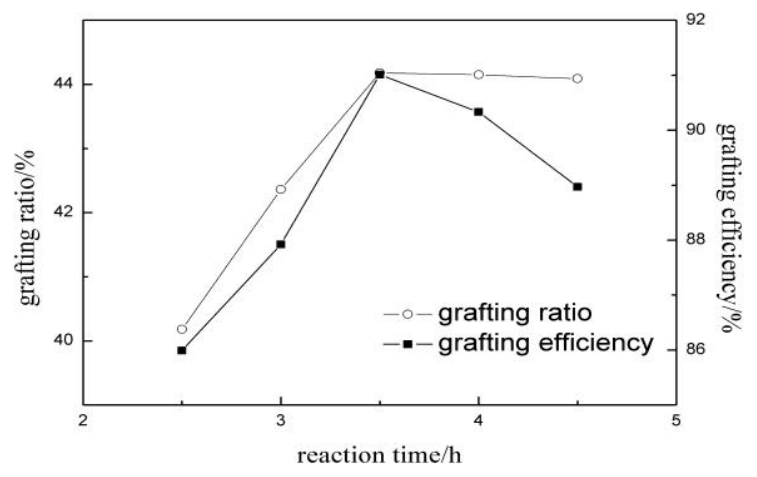

Fig. 7 Effect of the reaction time on grafting ratio

\section{Effect of the Reaction Time}

We studied the effect of reaction time on grafting ratio, as shown in Fig.7. We could see that the grafting ratio increased rapidly with the reaction time. But after the reaction time exceeded 3.5 hours, the grafting ratio decreased. So the reaction time was optimized as 5 hours. Increasing the reaction time can promote the grafting reaction of maleic anhydride and starch. To increase the reaction time, the grafted starch decreased and the side reactions, leading to decreased the grafting ratio.

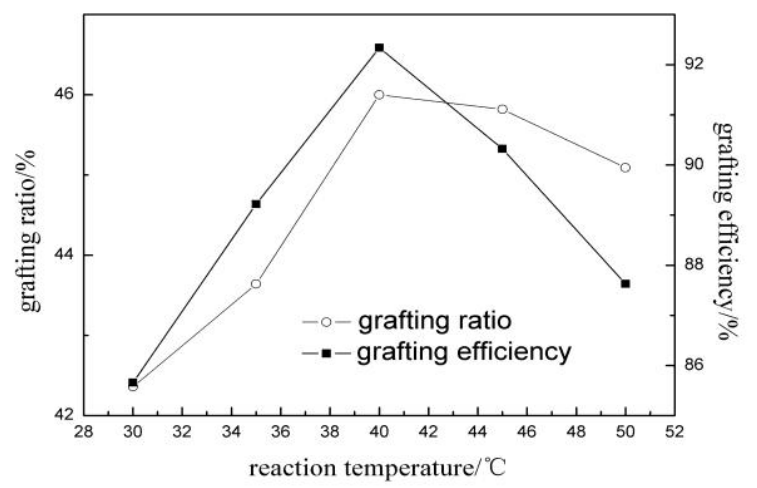

Fig. 8 Effect of the reaction tempertature on grafting ratio

\section{Effect of the Temperature Time}

Effect of reaction temperature on the grafting ratio and grafting efficiency are shown in Fig. 8 . The grafting ratio of starch increased with the reaction temperature increased initially. The grafting ratio reached the maximum value at $40{ }^{\circ} \mathrm{C}$. Then, the grafting ratio decreased with the increase of the reaction temperature. The reaction temperature is increased, so that maleic anhydride can more easily enter inside starch. But the temperature is too high, will lead to the occurrence of side reactions, leading to decline the grafting ratio.

\section{Orthogonal Experiment}

According to the results of single factor experiment, with the grafting ratio as indexes, the ratio of starch dosage and MAH dosage, solvent water dosage, reaction temperature, reaction time as factors, design orthogonal tests, and results of experiments were analyzed by using maximum difference method. The levels and factors of experiment were shown in Tab. 1, the orthogonal experiment result were shown in Tab. 2. 
Tab. 1 Levels of factors

\begin{tabular}{ccccc}
\hline & $\mathrm{A}$ & $\mathrm{B}$ & $\mathrm{C}$ & $\mathrm{D}$ \\
\cline { 2 - 5 } Levels & $\begin{array}{c}\text { ratio of starch } \\
\text { dosage and } \\
\text { MAH dosage }\end{array}$ & $\begin{array}{c}\text { solvent water } \\
\text { dosage } \\
(\mathrm{mL} / \mathrm{g})\end{array}$ & $\begin{array}{c}\text { reaction time } \\
(\mathrm{h})\end{array}$ & $\begin{array}{c}\text { reaction } \\
\text { temperature }\end{array}$ \\
1 & $3: 2$ & 1.0 & 3.5 & $\left({ }^{\circ} \mathrm{C}\right)$ \\
2 & $3: 2.5$ & 1.5 & 4.0 & 35 \\
3 & $3: 3$ & 2.0 & 4.5 & 40 \\
\hline
\end{tabular}

Tab. 2 Results of $\mathrm{L}_{9}$ (34) orthogonal experiment

\begin{tabular}{cccccc}
\hline No. & A & B & C & D & G\% \\
\hline 1 & 1 & 1 & 1 & 1 & 42.00 \\
2 & 1 & 2 & 2 & 2 & 45.40 \\
3 & 1 & 3 & 3 & 3 & 44.60 \\
4 & 2 & 1 & 2 & 3 & 46.50 \\
5 & 2 & 2 & 3 & 1 & 46.67 \\
6 & 2 & 3 & 1 & 2 & 48.67 \\
7 & 3 & 1 & 3 & 2 & 49.86 \\
8 & 3 & 2 & 1 & 3 & 43.29 \\
9 & 3 & 3 & 2 & 1 & 47.14 \\
\hline
\end{tabular}

Tab. 3 Analysis of variance

\begin{tabular}{cccccc}
\hline factors & & A & B & C & D \\
\hline \multirow{2}{*}{$\mathrm{G} \%$} & $\mathrm{~K} 1$ & 131.0 & 141.36 & 133.96 & 135.81 \\
& $\mathrm{~K} 2$ & 144.84 & 135.36 & 142.04 & 143.93 \\
$\mathrm{~K} 3$ & 140.29 & 140.41 & 141.13 & 137.39 \\
\multicolumn{2}{c}{ optimized condition of } & 13.84 & 6.00 & 8.08 & 8.12 \\
\multicolumn{2}{c}{ reaction } & $\mathrm{A} 2$ & $\mathrm{~B} 1$ & $\mathrm{C} 2$ & $\mathrm{D} 2$
\end{tabular}

From the analysis above, we could see the influence of the four factors from strong to weak was ordered as: $\mathrm{A}>\mathrm{D}>\mathrm{C}>\mathrm{B}$. And the optimized condition of reaction was: the reaction temperature was $35 \mathrm{C}$, the reaction time was $4 \mathrm{~h}$, the ratio of starch dosage and MAH dosage was 3:2, the amount of solvent water was $1 \mathrm{~mL} / \mathrm{g}$.

\section{Verification Experiment}

Validation experiment were performed with optimized condition of reaction, the results were shown in Table 4.

Tab 4 Verification experiment

\begin{tabular}{ccc}
\hline No. & G\% & GE\% \\
\hline 1 & 46.52 & 90.12 \\
2 & 47.38 & 87.64 \\
3 & 47.91 & 91.59 \\
average & 47.27 & 89.78 \\
\hline
\end{tabular}

\section{Conclusions}

Maleic anhydride was grafted onto the starch backbone; the optimal process conditions for 
maleic anhydride grafted starch were as follows: the reaction temperature was $35 \mathrm{C}$, the reaction time was $4 \mathrm{~h}$, the ratio of starch dosage and MAH dosage was 3:2, the solvent water dosage was 1 $\mathrm{mL} / \mathrm{g}$, the grafting ratio and grafting efficiency were $47.27 \%$ and $89.78 \%$ respectively.

\section{Acknowledgement}

Financial support from the Yili vocational and technology college Science Fund (yzyxm2016001) is acknowledged.

\section{References}

[1] Y. Li, H.B. Kang, Optimizing preparation conditions of hydrolyzed octenyl succinic anhydride modified eat Starc, Academic Periodical of Farm Products Processing. 20 (2011) 42-44.

[2] S.D. Zhang, X. Zhang, Y. Tang, H.X. Huang, B.Y Yan, Preparation and properties of oxalate starch, Chemical Research and Application. 21 (2009) 481-485.

[3] X. Qiao, Sort and application of modified starch, Dyestuff and Staining, 47 (2010) 44-47.

[4] Y. Jiang, The utilizing of okara in the food Industry, Grain Technology and Economy. 37 (2012) $39-41$.

[5] H.Y. Hu, Z.B. Tan, J. Shi, Z.Q. Huang, Advances of starch sodium dodecenylsuccinate, Popular science \& technology. 14 (2012) 59-60.

[6] J.W He, Y. Hong. Applications of physicochemical methods in research of modified starch, Cereals \& Oils. 10 (2012) 6-8. 